\title{
Identification and analysis of glutathione S-transferase gene family in sweet potato reveal divergent GST-mediated networks in aboveground and underground tissues in response to abiotic stresses
}

\author{
Na Ding ${ }^{1,2+}$, Aimin Wang ${ }^{1+}$, Xiaojun Zhang ${ }^{1,2}$, Yunxiang Wu' ${ }^{1}$, Ruyuan Wang ${ }^{1}$, Huihui Cui ${ }^{2}$, Rulin Huang ${ }^{2}$ \\ and Yonghai Luo ${ }^{1,2^{*}}$
}

\begin{abstract}
Background: Sweet potato, a hexaploid species lacking a reference genome, is one of the most important crops in many developing countries, where abiotic stresses are a primary cause of reduction of crop yield. Glutathione S-transferases (GSTS) are multifunctional enzymes that play important roles in oxidative stress tolerance and cellular detoxification.

Results: A total of 42 putative full-length GST genes were identified from two local transcriptome databases and validated by molecular cloning and Sanger sequencing. Sequence and intraspecific phylogenetic analyses revealed extensive differentiation in their coding sequences and divided them into eight subfamilies. Interspecific phylogenetic and comparative analyses indicated that most examined GST paralogs might originate and diverge before the speciation of sweet potato. Results from large-scale RNA-seq and quantitative real-time PCR experiments exhibited extensive variation in gene-expression profiles across different tissues and varieties, which implied strong evolutionary divergence in their gene-expression regulation. Moreover, we performed five manipulated stress experiments and uncovered highly divergent stress-response patterns of sweet potato GST genes in aboveground and underground tissues.

Conclusions: Our study identified a large number of sweet potato GST genes, systematically investigated their evolutionary diversification, and provides new insights into the GST-mediated stress-response mechanisms in this worldwide crop.
\end{abstract}

Keywords: Sweet potato, Glutathione S-transferase, Evolutionary diversification, Regulatory mechanism, Abiotic stresses

\section{Background}

Gene duplication is one of central research themes in evolutionary biology through which new genetic materials for phenotypic innovations are generated. After duplication, the duplicated genes may functionally diversify in protein

\footnotetext{
*Correspondence: yonghailuo@foxmail.com; yhluo@jsnu.edu.cn

${ }^{\dagger}$ Equal contributors

${ }^{1}$ Plant Functional Genomics, School of Life Sciences, Jiangsu Normal University, 101 Shanghai Road, Tongshan New District, Xuzhou City, Jiangsu Province 221116, China

${ }^{2}$ Center for Molecular Cell and Systems Biology, College of Life Sciences, Fujian Agriculture and Forestry University, Fuzhou City, Fujian Province 350002, China
}

property and/or spatiotemporal gene-expression pattern, and eventually lead to distinct evolutionary consequences: non-functionalization, subfunctionalization, or neofunctionalization $[1,2]$. A contemporary gene family in a specific species represents a set of extant genes derived from a single ancestor, as an evolutionary consequence of whole genome and/or gene duplications. Systematic investigation of a gene family, in divergence of both protein-coding sequence and gene-expression profiles, would advance our understandings towards its origin and evolution and provide important insights into gene function and application $[3,4]$. 
Glutathione S-transferases (GSTs, EC 2.5.1.18) are a family of multifunctional dimeric enzymes, which widely function in cellular detoxification of xenobiotic and endobiotic compounds by conjugating the tripeptide glutathione (GSH; $\gamma$-L-glutamyl-L-cysteinyl-L-glycine) to various substrates [5]. GST genes have been ubiquitously found in eukaryotes and prokaryotes, and well-studied across plants, animals, fungi, and bacteria [6]. In higher plants, GSTs have been classified into eight typical subfamilies, including Phi (GSTF), Tau (GSTU), Lambda (GSTL), dehydroascorbate reductase (DHAR), Theta (GSTT), Zeta (GSTZ), elongation factor 1 gamma (EF1By), and tetrachlorohydroquinone dehalogenase (TCHQD) [7, 8]. Amongst these subfamilies, Phi, Tau, Lambda, and DHAR are plant-specific [5]. A typical GST protein contains two conserved active sites: one is a GSH-binding site (G-site) in the $\mathrm{N}$-terminal domain, and the other is a C-terminal co-substrate-binding domain $(\mathrm{H}$ site). G-site is specific for GSH and mainly affects the catalytic function, whereas the $\mathrm{H}$-site contributes to the conjunction of specific substrate $[8,9]$.

It has been functionally demonstrated that plant GST genes are widely involved in the detoxification of herbicides, as well as in response to biotic and abiotic stresses [10]. GST genes could respond to a wide range of stress treatments such as ozone, hydrogen peroxide, plant hormone, heavy metal, heat shock, wounding, and dehydration [11-13]. Among the eight GST subfamilies, the functions of Tau and Phi subfamily members are the most widely studied. For example, the expression of AtGSTU19 could be induced in the arid environment [14-16], and AtGSTF10 is involved in salt stress and BAK1-mediated spontaneous cell death signaling pathway [17]. In addition, genes in these two subfamilies are involved in the transport and metabolism of secondary compounds [18-20]. For example, the maize $B z 2$ gene, the petunia An9 gene, and the Arabidopsis TT19 gene function in anthocyanin transport and vacuolar sequestration [19, 21, 22]. GST genes in other subfamilies are also multifunctional: some GSTS in the Zeta subfamily are involved in tyrosine metabolism [23, 24], some in the DHAR subfamily could catalyze the metabolism of ascorbic acid [8, 25], some in the Lambda subfamily can be used as antioxidant and selectively bound to flavonol [26], and members of the EF1By subfamily mainly function as glutathione peroxidases, which protect cells from interference and damage by oxide [27, 28].

Sweet potato [Ipomoea batatas (L.) Lam.] is one of the most important crops in the world because it provides an indispensable caloric source for human beings, especially those living in Sub-Saharan Africa and East Asia [29]. Because sweet potato can adapt and grow well in diverse harsh environments, it ensures food supply and safety in developing countries. However, advances in fundamental research for this outcrossing hexaploid crop
$(2 n=6 x=90)$ are highly limited because of its complex genetic composition, which has been thought to experience multiple whole-genome duplications during speciation [30-32]. To date, no high-quality reference genome sequence for sweet potato is available to date. Therefore, investigations of genome-wide gene duplications and their evolution in sweet potato remain a challenge. Whole transcriptome sequencing (i.e., RNA-seq) provides a valuable alternative to whole genome sequencing for gene mining and functional characterization [33, 34]. In particular, the third-generation sequencing technologies have enabled us to obtain long-read or full-length transcriptomes, which allows collection of large-scale long-read transcripts with complete coding sequences and characterization of gene families [35-38]. In the present study, we identified 42 putative full-length and 19 partial GST genes from our high-quality transcriptome databases in sweet potato and investigated their divergence in coding sequences, geneexpression profiles, and biological functions in response to multiple abiotic stresses. Our study serves as the first case involving the characterization of a transcriptome-wide gene family in sweet potato, which is a genetically complex organism lacking high-quality reference genome sequences. Our results reveal new insights into distinct regulatory mechanisms in aboveground and underground tissues in GST-mediated response to abiotic stresses in sweet potato.

\section{Methods}

\section{Generation of high-quality transcriptome databases in sweet potato by second- and third-generation RNA sequencing technologies}

Previously, we reported 53,861 high-quality long-read transcripts for sweet potato, which were generated by a combination of Illumina second-generation and PacBio third-generation sequencing technologies [39]. In this study, we further assembled the obtained Illumina second-generation reads together with 53,861 long-read transcripts to generate a combined transcriptome database (named as DB12; transcript number: 200,752). DB12 was generated from a single variety of Xushu18, an elite sweet potato variety in China, with the aim of reducing the transcriptome complexity and improving the accuracy of the transcript assembly. In another ongoing project, we sequenced the transcriptomes of mature tuberous root of each of 77 sweet potato varieties (36 purple-flesh and 41 non-purple-flesh) using the Illumina second-generation sequencing technology. All Illumina short reads from the 77 varieties were pooled for a transcriptome assembly (namely, DB77), which generated 305,505 transcripts. The transcriptome databases of DB12 and DB77 are available upon request. Sampled tissues that were used for the generation of DB12 and DB77 were illustrated in Additional file 1: Figure S1. 


\section{Identification of GST genes from the transcriptome databases DB12 and DB77}

We performed local BLAST and domain search for genes containing GST N-terminal and C-terminal domain in the transcriptome databases DB77 and DB12. First, we retrieved Arabidopsis GST protein sequences from website (http://www.arabidopsis.org/). The obtained Arabidopsis GST protein sequences were used as the query to perform BLAST searches against DB12 and DB77. A cut-off E-value $\left(\leq \mathrm{e}^{-3}\right)$ was applied to filter the homologous transcripts. Secondly, the obtained transcript sequences from DB12 and DB77 databases were translated and analyzed by the PFAM program (http://pfam.xfam.org) to examine the presence of the GST domains. Furthermore, we removed the transcripts encoding short proteins with less than 120 amino acids and confirmed the presence of GST domains by analyzing the deduced proteins of filtered transcripts in the NCBI Conserved Domain Database (CDD, http:// www.ncbi.nlm.nih.gov/Structure/cdd/wrpsb.cgi?). The following parameters were used in the CDD analysis: E-value, 0.01; maximum number of hits, 500; and the result mode, Concise. The transcripts which did not contain a complete GST N-terminal or C-terminal domain in CDD analysis were eliminated. Finally, we removed one redundant sequence if two transcripts had the identity of amino acids equal to or larger than $97 \%$ and obtained a final gene list. The pairwise identity matrix of 43 full-length GSTs was generated by the software BioEdit [40].

\section{Molecular cloning and Sanger sequencing of GST genes in a sweet potato variety}

A pooled sample (including 8 tissues of shoot, young leaf, mature leaf, stem, fibrous root, initial tuberous toot, expanding tuberous root, and mature tuberous root) was collected from a single sweet potato variety (Nanzishu8) that was randomly selected from DB77 varieties. Total RNA was isolated from the pooled sample using TRIzol and cDNA was synthesized by reverse transcription Kit (ProbeGene, China). To clone the transcriptome-derived GST genes, gene-specific primers were designed used for PCR amplification using the synthesized cDNA as templates (Additional file 2: Table S1). Amplified fragments were cloned into the vector pUC57 and subjected to Sanger sequencing. The obtained sequences were compared to the corresponding transcripts obtained from the transcriptome databases and the polymorphism data are summarized in Table 1.

\section{Construction of phylogenetic trees and motif analysis}

The protein sequences of identified sweet potato GSTs were aligned and phylogenetic trees were created using MEGA 7.0 (Molecular Evolutionary Genetics Analysis) program [41]. Alignments were performed using the Muscle program with default parameters, and the results were then subjected to construct unrooted phylogenetic trees using both the Maximum Likelihood (ML) method and the Neighbor-Joining (NJ) method, where the bootstrap analyses were carried out with 1000 replicates. The online MEME program (http://meme-suite.org/) was used for motif analysis. We set the maximum number of motifs to ten and other parameters were set to default.

\section{Analysis of gene-expression profiles}

First, we investigated the variation of gene-expression profiles in the tuberous roots of the DB77 varieties. For each GST gene, representative transcripts in DB77 were identified and the FPKM values of representative transcripts were extracted for a clustering analysis using the Cluster 3.0 program. The parameters of clustering analysis were as follows: all of the data were adjusted by log transformation and hierarchical clustering analysis was chosen as calculating method and complete linkage as clustering method. At last, the heat map was shown by Java TreeView.

Second, we surveyed the expression pattern of the GST genes in 8 different tissues of one purple-flesh (Xuzi3) and one non-purple-flesh (Yan252) sweet potato variety. The 8 tissues included shoots, young leaves, mature leaves, stems, fibrous roots, initial tuberous roots, expanding tuberous roots, and mature tuberous roots (Additional file 1: Figure S1). High-throughput RNA sequencing was performed and a transcriptome database (named as DB16) was assembled from pooled RNA-seq data of 16 samples. Representative transcripts of GST genes from DB16 were identified and FPKM values of representative transcripts were extracted from DB16 for a clustering analysis using the Cluster 3.0 program. In both cases, we determined the representative transcripts in a database by BLASTn search with following criteria: coverage of the first alignment larger than $40 \%$ of the investigated gene and identity of the aligned sequences larger than $97 \%$.

\section{Quantitative RT-PCR (RT-qPCR) experiments}

To validate the GST gene-expression profiles observed in the transcriptomic experiments described above, we performed RT-qPCR analysis of 9 GST genes using the same tissue samples in DB16. The examined genes and primer sequences are listed in Additional file 2: Table S1. Total RNA was extracted using TRIzol reagents and the provided protocol (Invitrogen, USA). For each sample, three technical replicates of RT-qPCR were done. The expression of each gene in different samples was normalized with the expression of an internal control gene, $A R F$, to ensure the equal amount of cDNA used for individual reactions. The mRNA levels for each gene in different tissue samples were calculated using the $\Delta \Delta C T$ method. The relative gene expression levels in 16 tissue samples 
Table 1 Comparison of putative coding sequences of 43 GST genes obtained from transcriptomes (CDS1) and laboratory cloning (CDS2)

\begin{tabular}{|c|c|c|c|c|c|c|c|}
\hline Gene name & Length of CDS1 (bp) & Length of CDS2 (bp) & $\Delta$ Length (bp) & Type of Polymorphism & Alignment length (bp) & Identical (bp) & Identity (\%) \\
\hline |bDHAR| & 642 & 642 & 0 & SNP & 642 & 638 & 99.38 \\
\hline IbDHAR2 & 813 & 813 & 0 & SNP & 813 & 808 & 99.39 \\
\hline IbEF|Bү| & 1260 & 1270 & 10 & SNP \& InDel & 1272 & 1245 & 97.88 \\
\hline IbEF|Bү2 & 1260 & 1260 & 0 & SNP & 1260 & 1253 & 99.44 \\
\hline IbEF1Bץ3 & 1293 & 1260 & -33 & SNP \& InDel & 1261 & 1155 & 91.59 \\
\hline IbGSTFI & 675 & 675 & 0 & SNP & 675 & 668 & 98.96 \\
\hline IbGSTF2 & 642 & 739 & 97 & SNP \& InDel & 647 & 264 & 40.80 \\
\hline IbGSTF3 & 645 & 643 & -2 & SNP & 643 & 638 & 99.22 \\
\hline IbGSTLI & 711 & 711 & 0 & SNP & 711 & 710 & 99.86 \\
\hline IbGSTL2 & 705 & 705 & 0 & SNP & 705 & 694 & 98.44 \\
\hline IbGSTL3 & 810 & 810 & 0 & SNP & 810 & 804 & 99.26 \\
\hline |bGSTT| & 708 & 708 & 0 & SNP & 708 & 691 & 97.60 \\
\hline IbGSTT2 & 708 & 708 & 0 & SNP & 708 & 700 & 98.87 \\
\hline IbGSTUI & 675 & 673 & -2 & SNP & 673 & 667 & 99.11 \\
\hline IbGSTU2 & 666 & 666 & 0 & N.A. & 666 & 666 & 100.00 \\
\hline IbGSTU3 & 660 & 661 & 1 & SNP \& InDel & 661 & 613 & 92.74 \\
\hline IbGSTU4 & 690 & 690 & 0 & N.A. & 690 & 690 & 100.00 \\
\hline IbGSTU5 & 684 & 684 & 0 & SNP & 684 & 679 & 99.27 \\
\hline IbGSTU6 & 672 & 663 & -9 & SNP & 653 & 640 & 98.01 \\
\hline IbGSTU7 & 714 & 728 & 14 & SNP \& InDel & 729 & 688 & 94.38 \\
\hline IbGSTU8 & 672 & 663 & -9 & SNP & 663 & 650 & 98.04 \\
\hline IbGSTU9 & 714 & 714 & 0 & SNP & 714 & 707 & 99.02 \\
\hline IbGSTUIO & 660 & 738 & 78 & SNP \& InDel & 738 & 652 & 88.35 \\
\hline |bGSTUI| & 684 & 661 & -23 & SNP \& InDel & 651 & 616 & 94.62 \\
\hline IbGSTUI2 & 675 & 675 & 0 & SNP & 675 & 673 & 99.70 \\
\hline IbGSTUI3 & 672 & 672 & 0 & SNP & 672 & 658 & 97.92 \\
\hline IbGSTU|4 & 672 & 672 & 0 & SNP & 672 & 637 & 94.79 \\
\hline IbGSTUI5 & 687 & 689 & 2 & SNP \& InDel & 689 & 674 & 97.82 \\
\hline IbGSTUI6 & 672 & 660 & -12 & SNP & 660 & 646 & 97.88 \\
\hline IbGSTUI7 & 669 & 669 & 0 & SNP & 669 & 656 & 98.06 \\
\hline IbGSTUI8 & 666 & 663 & -3 & SNP & 663 & 659 & 99.40 \\
\hline IbGSTUI9 & 729 & 714 & -15 & SNP \& InDel & 713 & 691 & 96.91 \\
\hline IbGSTU2O & 678 & 673 & -5 & SNP & 673 & 664 & 98.66 \\
\hline IbGSTU2| & 684 & 684 & 0 & SNP & 684 & 676 & 98.83 \\
\hline IbGSTU22 & 687 & 687 & 0 & SNP & 687 & 667 & 97.09 \\
\hline IbGSTU23 & 702 & 702 & 0 & SNP & 702 & 699 & 99.57 \\
\hline IbGSTU24 & 669 & 665 & -4 & SNP & 665 & 657 & 98.80 \\
\hline IbGSTU25 & 687 & 680 & -7 & SNP & 680 & 671 & 98.68 \\
\hline IbGSTU26 & 744 & 837 & 93 & SNP \& InDel & 837 & 739 & 88.29 \\
\hline IbGSTU27 & 672 & 662 & -10 & SNP \& InDel & 665 & 633 & 95.19 \\
\hline IbGSTZI & 900 & 894 & -6 & SNP \& InDel & 900 & 876 & 97.33 \\
\hline IbGSTZ2 & 672 & 672 & 0 & SNP & 672 & 668 & 99.41 \\
\hline IbGSTZ3 & 672 & 666 & -6 & SNP \& InDel & 666 & 659 & 98.95 \\
\hline
\end{tabular}


were further normalized with the expression in shoot of non-purple-flesh sweet potato.

\section{Response of GST genes to abiotic stresses}

To investigate the expression patterns of GST genes under normal growth condition and abiotic stresses, shoot cuttings of sweet potato were cultivated in $1 \%$ o Hoagland's hydroponic medium for 7 days and then transferred to hydroponic boxes containing 5.0\% hydrogen peroxide $\left(\mathrm{H}_{2} \mathrm{O}_{2}\right), 200 \mu \mathrm{M}$ cupric sulfate $\left(\mathrm{CuSO}_{4}\right)$, $40 \mu \mathrm{M}$ arsenic solution $\left(\mathrm{As}_{2} \mathrm{O}_{3}\right), 1.5 \mathrm{mM}$ cadmium carbonate $\left(\mathrm{CdCO}_{3}\right)$ and $2 \mathrm{mM}$ zinc vitriol $\left(\mathrm{ZnSO} 4 \cdot 7 \mathrm{H}_{2} \mathrm{O}\right)$, respectively. Cultivation in $1 \%$ Hoagland's hydroponic medium was used as control. Each treatment consisted of eight duplicates of shoot cuttings (classified into two subgroups, each group had four duplicates). After $48 \mathrm{~h}$, the aboveground (including shoots, young leaves, and mature leaves) and underground (adventitious roots) tissues were collected, respectively, from each subgroup. All samples were frozen immediately with liquid nitrogen and stocked in a $-80{ }^{\circ} \mathrm{C}$ freezer. Total RNA extraction, cDNA synthesis, and quantitative real-time PCR were performed as described above.

\section{Results \\ Identification and validation of transcriptome-wide GST genes in sweet potato}

We searched the two transcriptome databases (DB12 and DB77) using BLAST to identify candidate GST transcripts. The presence of conserved GST N-terminal domain (i.e., PFAM domain PF02798) in each transcript was confirmed in the NCBI Conserved Domain Database, and redundant transcripts were removed. A total of 62 putative non-redundant GST genes were identified (Additional file 3: Table S2). Domain structure analysis suggested that 43 of these had complete N- and C- terminal domains (i.e., full-length GST genes) and 19 had only a N-terminal or C-terminal domain (i.e., partial GST genes). Sequence comparisons indicated that the pairwise identity of the coding sequences (CDS) of the full-length GSTs ranged from 0.127 to 0.979 , whereas the pairwise identity of the deduced amino acids ranged from 0.050 to 0.951 (Additional file 4: Table S3).

Erroneous transcripts could be generated in highthroughput transcriptome sequencing projects due to assembly errors. To validate the transcriptome-derived sequences, we designed gene-specific primers and cloned the predicted coding sequences of all 43 full-length GST genes from a single sweet potato variety (Nanzishu8) that were randomly picked up from the DB77 varieties. cDNA sequences of all 43 GST genes were successfully cloned from Nanzishu8 and all but one (i.e., IbGSTF2) sequences were highly homologous to the corresponding GSTs that were identified in the transcriptome databases
(Table 1). In contrast to the transcriptome-derived IbGSTF2 sequence, the cloned fragment shared only $40.80 \%$ identity. This was likely attributable to incorrect transcriptome assembly or molecular cloning. We also found relatively low identity between transcriptomederived and cloned sequences of IbGSTU10, which was actually due to the existence of a 78-bp insertion in the cloned copy. A similar situation was found for IbGSTU26. The other 40 GST sequences cloned from Nanzishu8 shared high identity (i.e., > 90\%) with those derived from our transcriptome databases (Table 1). Considering the presence of genetic variation among sweet potato varieties, we concluded that except for IbGSTF2, the other 42 GST genes identified in our transcriptome databases were indeed present in the sweet potato genome.

\section{Phylogenetic and comparative analyses of GST genes in and beyond the sweet potato species}

Using phylogenetic analysis, we classified the 42 fulllength GST proteins into seven major clades (i.e., subfamily): Tau (27 members), Phi (2), Theta (2), Zeta (3), EF1BY (3), Lambda (3), and DHAR (2) (Fig. 1a, Table 2, Additional file 5: Figure S2). A total of 10 putative motifs were predicted by the program MEME across all members. The arrangement of motifs within each subfamily was comparable, but diverse among different subfamilies (Fig. 1b). It has been reported that most of GST proteins contain motifs 1,3 , and 6 , which jointly constitute the basis of N-terminal domain. Our analysis revealed a consistent result that the three motifs were present in almost all GST proteins. Motif 6 is found in members of Tau, Phi, Theta, EF1B $\gamma$, and DHAR subfamilies; motifs 3 and 5 are specific to the Tau subfamily; Motif 7 is specific to $\mathrm{EF} 1 \mathrm{~B} \gamma$ members; motif 8 and 9 are present in the Theta subfamily only; whereas motif 10 is found in the $\mathrm{EF} 1 \mathrm{~B} \gamma$ and Theta subfamilies. We further analyzed the phylogenetic relationship among the GST proteins in sweet potato, I. trifida, I. nil, and A. thaliana (Additional file 6: Table S4). Figure 2 shows that 42 sweet potato GST proteins are clustered into eight subfamilies (Fig. 2 and Additional file 7: Figure S3) In all but TCHQD subfamilies, genes derived from each of the four species were included. In the TCHQD subfamily, no GSTs were identified in sweet potato and I. trifida. Overall, the majority of GST members of sweet potato, $I$. trifida, and $I$. nil were interspersed (i.e., show a mosaic pattern) within the phylogenetic tree, whereas most $A$. thaliana members were clustered into isolated subclades. These findings imply that most gene duplication events occurred probably before the speciation of sweet potato and after the divergence from $A$. thaliana.

Furthermore, we compared the number and distribution of GST genes across sweet potato and eight other 


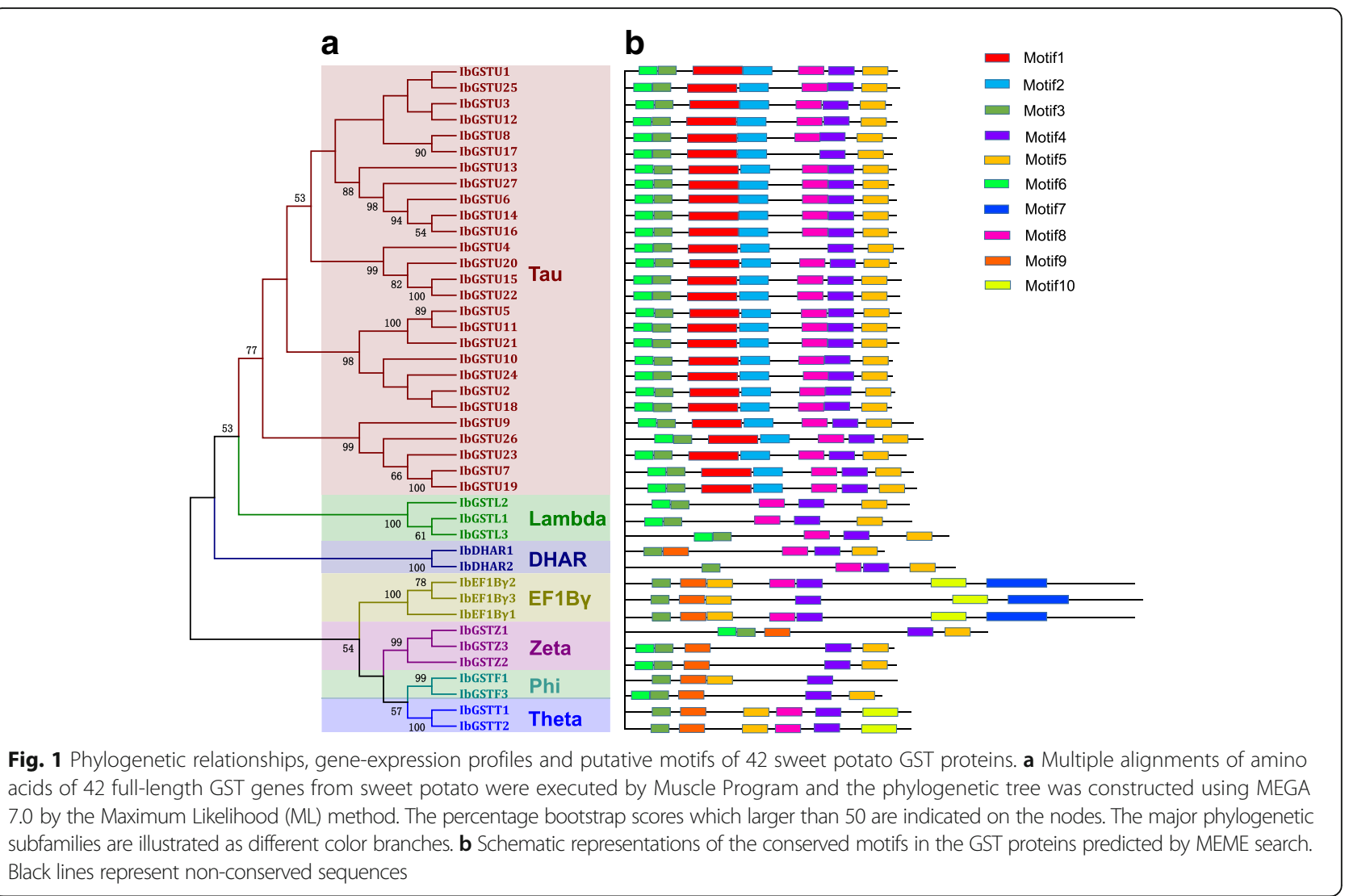

plant species, including Arabidopsis thaliana, Oryza sativa, Hordeum vulgare L., Populus L., Zea mays, Glycine max, Ipomoea trifida, and Ipomoea nil (Table 2). The number of sweet potato GST genes (even plus 19 partial GSTs) identified in this study is less than those of I. nil, O. sativa, $H$. vulgare, P. trichocarpa, and G. max. This indicates that the collection of GST genes in our study was likely incomplete, and it might be hard to identify all members of a gene family from transcriptome databases. Amongst these, the Tau subfamily constitutes a biggest subfamily, which accounts for more than half of the total number of GST genes in each plant. In sweet potato, we identified 27 out of the 42 GST genes as Tau members. The Phi subfamily is the second-largest class of GSTs in various plants, and there are 13, 17, 21, 9, 7, and 17 in Arabidopsis, rice, barley, poplar, maize, and soybean. However, we identified that the Phi subfamily of sweet potato has only 3 members, which is less than the other species. Similarly, the number of Phi members in I. trifida and I. nil was 4 and 6, respectively. Although previous studies have shown that the Zeta and Theta subfamilies represent the oldest GST genes, their numbers in plants are less than those of plant specific subfamilies, Tau and Phi. These data suggest that members

Table 2 Number of different subfamilies of GST genes in nine species

\begin{tabular}{|c|c|c|c|c|c|c|c|c|c|}
\hline GST gene subfamily & Tau & Phi & Theta & Zeta & EF1BY & Lambda & DHAR & TCHQD & Total \\
\hline Arabidopsis thaliana & 28 & 13 & 3 & 2 & 2 & 3 & 3 & 1 & 55 \\
\hline Oryza sativa & 52 & 17 & 1 & 4 & 2 & 3 & 2 & 1 & 82 \\
\hline Hordeum vulgare & 50 & 21 & 1 & 5 & 2 & 2 & 2 & 1 & 84 \\
\hline Populus trichocarpa & 58 & 9 & 2 & 2 & 3 & 3 & 3 & 1 & 81 \\
\hline Zea mays & 27 & 7 & 0 & 3 & 0 & 0 & 0 & 0 & 37 \\
\hline Glycine max & 63 & 17 & 3 & 3 & 0 & 8 & 0 & 0 & 94 \\
\hline Ipomoea trifida & 24 & 4 & 2 & 3 & 4 & 4 & 2 & 0 & 43 \\
\hline Ipomoea nil & 48 & 6 & 4 & 7 & 4 & 3 & 2 & 1 & 74 \\
\hline Ipomoea batatas & 27 & 3 & 2 & 3 & 3 & 3 & 2 & 0 & 43 \\
\hline
\end{tabular}




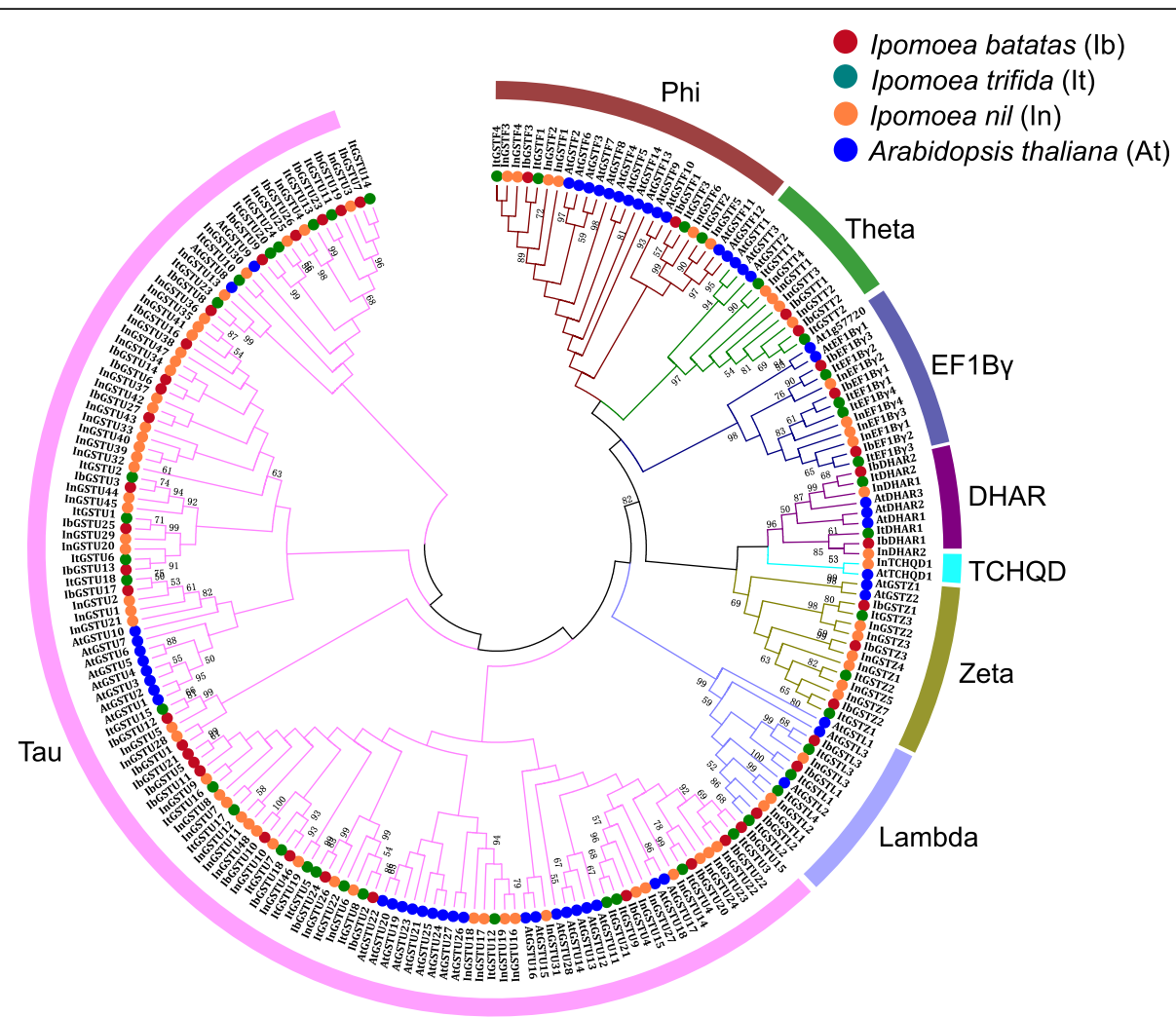

Fig. 2 Phylogenetic tree and subfamily classification of GST proteins from sweet potato, Ipomoea nil, Ipomoea trifida, and Arabidopsis thaliana. The unrooted tree was constructed based on multiple sequence alignment of full-length protein sequences using Muscle program by MEGA 7.0 by the Maximum Likelihood (ML) method. In total, 42, 43, 74, and 55 full-length GST proteins from sweet potato, I. nil, I. trifida, and A. thaliana, respectively, were included. The percentage bootstrap scores which $>50$ are indicated on the nodes. The major phylogenetic subfamilies are illustrated as different color branches

of the Tau and Phi subfamilies rapidly expanded in most plants and thus may play multifarious roles among various species.

To analyze the intron/exon structures of sweet potato GST genes, we aligned the coding sequences of 42 fulllength GST genes against the published sweet potato genomic contig sequences [32]. We found corresponding genomic sequences for 30 sweet potato GST genes. Subsequently, we identified their best-matched homologous genes of I. trifida and I. nil, and compared their gene structures (Fig. 3). Our data indicated that the examined GST genes shared similar intron/exon structures within each subfamily but were differed among subfamilies. Most of the genes in the Tau subfamily possessed a single intron, whereas those in Zeta contained up to 10 introns. Nevertheless, exceptions were observed. For instance, ItGSTU23 and ItGSTU6 had 4 and 3 introns, respectively (Fig. 3b). Notably, we found some sweet potato genes containing unusually large introns, such as IbGSTT2. Comparative analysis of IbGSTT2, InGSTT2, and ItGSTT2 indicated that each of these had 6 introns, whose sizes ranged from several hundreds to over 26,000 in base pairs (Fig. 3b). Taken together, our comparative analyses reinforce our viewpoint that most duplications and divergences of GST genes have likely occurred before the speciation of sweet potato (probably in a common ancestor of sweet potato, $I$. trifida, and I. nil).

\section{Gene expression profiles of the sweet potato GSTs}

To investigate the diversification of expression patterns of sweet potato GSTs, we performed two RNA-seq experiments. First, we investigated variations in GST gene expression in mature tuberous roots of 77 sweet potato varieties. In total, we identified homologous transcripts in DB77 that represented 35 of our GSTs. FPKM values of homologous transcripts were extracted and used for a clustering analysis. Figure 4 shows differences in expression patterns in the examined GSTs: (1) some GSTs were highly expressed across most or all 77 varieties; (2) some genes were with extremely low expression in almost all varieties; and (3) some other genes exhibited variations in expression among different varieties. For example, IbGSTL1, IbDHAR1, IbGSTU10, IbGSTU20, and IbGSTZ2 showed very high expression levels in all 77 sweet potato varieties, whereas IbGSTU8, IbGSTU21, and IbGSTU23 


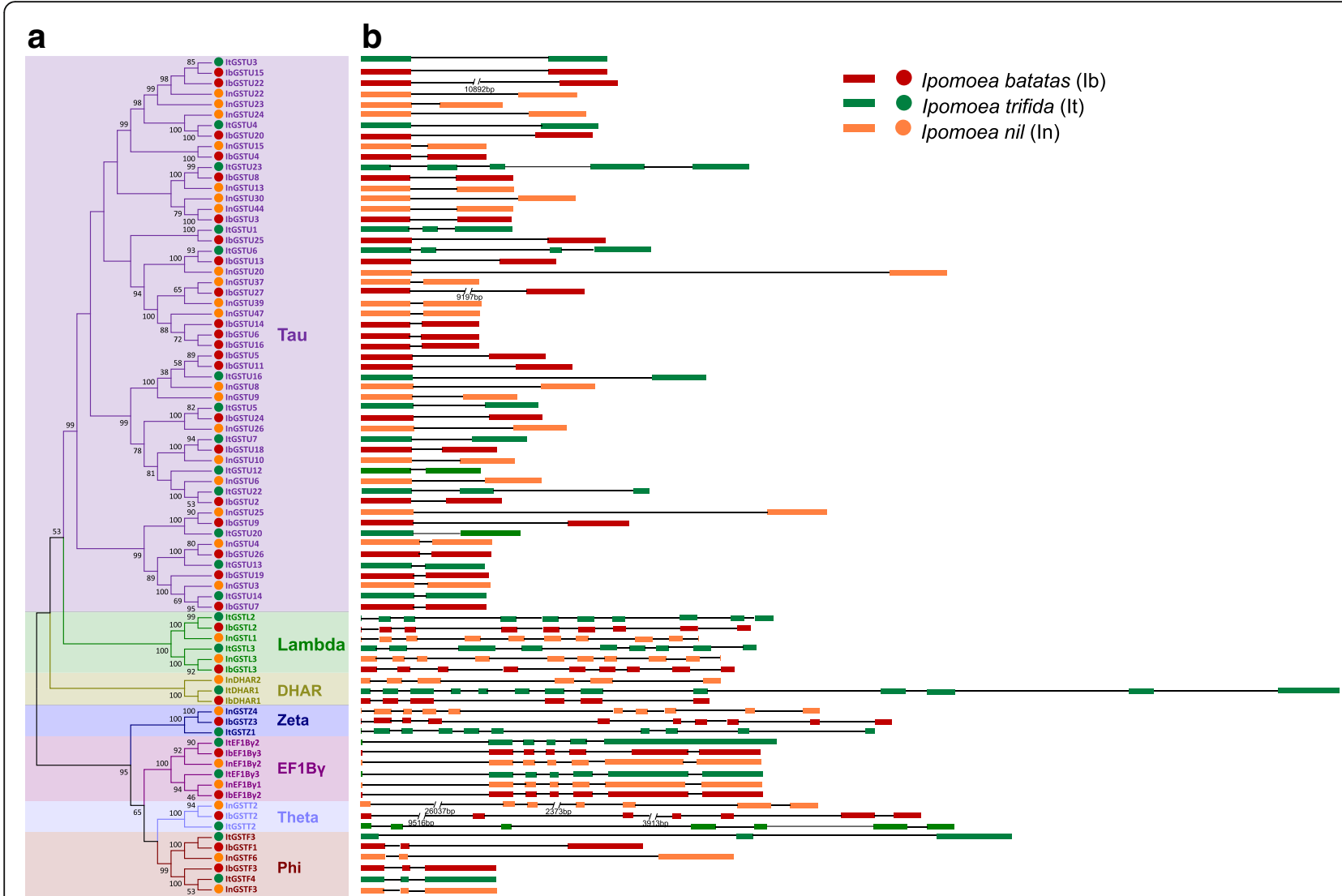

Fig. 3 Phylogenetic relationships (a) and intron-exon structures (b) of homologous GST genes in sweet potato, Ipomoea trifida, and Ipomoea nil. In (b), filled boxes represent exons, whereas lines represent introns

exhibited low expression levels in almost all 77 varieties. These results indicate that the expression of each GST gene in the tuberous root is influenced by genetic background, which varies among sweet potato cultivars. Notably, IbGSTF3 showed significantly higher expression in the tuberous roots of all purple-flesh sweet potato varieties than those in non-purple-flesh ones (Fig. 4, Fig. 7c).

Second, we surveyed the expression patterns of GST genes in 8 different tissues of one purple-flesh and one non-purple-flesh sweet potato varieties. We assembled a transcriptome database (named as DB16) from all RNAseq data of 16 samples and identified transcripts corresponding to 27 of our GST genes (Fig. 5a). Clustering analysis of the FPKM data revealed distinct expression patterns of these GST genes between aboveground and underground tissues. In the aboveground tissues, the gene expression data from two same tissues (e.g., Xuzi3$\mathrm{S}$ and Yan252-S) of purple-flesh and non-purple-flesh sweet potato varieties were clustered together, whereas in the underground tissues, data from the four tissues of either purple-flesh or non-purple-flesh sweet potato were grouped together (Fig. 6a). These data suggest that substantial divergence in regulation of the GST genes has occurred between aboveground and underground tissues of sweet potato. Furthermore, some GST genes demonstrated highly specific expression patterns. For example, IbGSTU1 showed relatively high expression only in shoot of purple-flesh sweet potato. On the other hand, we examined whether two phylogenetically close genes (i.e., with high similarity in coding sequences) exhibited highly mimic gene expression patterns across different tissues. We found that only a few phylogenetically close genes (e.g., IbGSTU23, and IbGSTU26) showed similar expression patterns, and the majority of genes did not (e.g., IbGSTF1 and IbGSTF3, IbGSTL1 and IbGSTL3) (Figs. 5a and 6a). These results imply that sequence divergence in coding sequences and regulatory regions of two duplicated genes was likely uncoupled.

In addition, we selected 9 GST genes and performed quantitative real-time PCR to confirm the gene expression patterns observed in the above described RNA-seq experiments. Overall, we found a high correlation in gene expression that was quantified using two approaches (Fig. 7a). That is, the expression patterns of 9 GST genes showed differences among varieties and tissues, which were in agreement with the data obtained by RNA-seq (Fig. $7 b-j)$. These results demonstrate the high level of reliability of our gene expression data. 


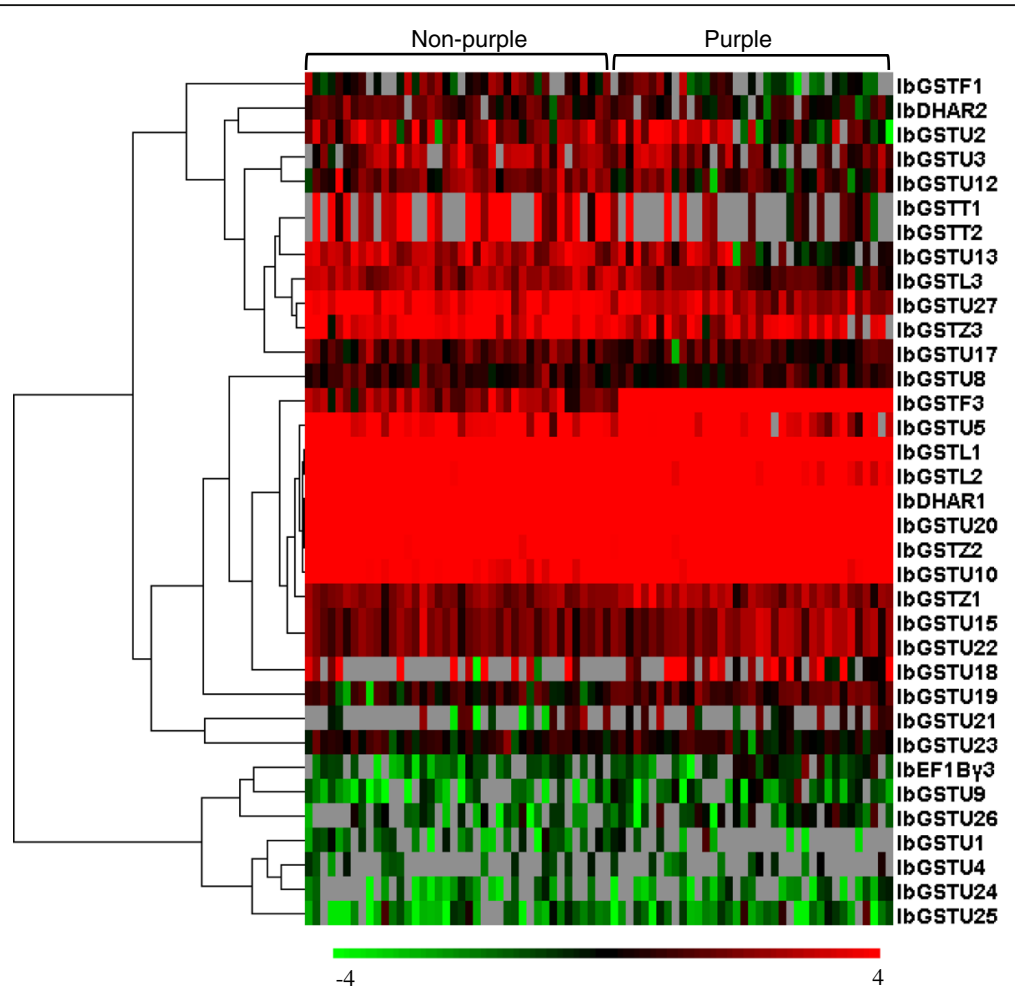

Fig. 4 Hierarchical clustering of gene-expression data of 35 GST genes in 77 varieties of sweet potato. Horizontal direction indicates 77 sweet potato varieties, including 36 purple-flesh and 41 non-purple-flesh ones. The longitudinal direction indicates 30 GST genes in sweet potato. The data were FPKM values extracted from DB77 (log2 transformed) and color scaled as shown in the bottom. Gray boxes mean FPKM=0

Roles of sweet potato GSTs in response to abiotic stresses Although sweet potato has the extraordinary ability to adapt to a wide spectrum of stresses, our understanding of its underlying molecular mechanisms is limited. Previous studies have revealed that GST genes are involved in plant responses to oxidative stresses and heavy metal toxicity $[42,43]$. In the present study, we examined the expression patterns of 42 sweet potato GST genes in response to stress treatments using $\mathrm{H}_{2} \mathrm{O}_{2}, \mathrm{Cu}, \mathrm{As}, \mathrm{Cd}$, and $\mathrm{Zn}$, respectively. Gene expression data of each GST were compared between the treated and untreated tissues to infer a stress-response pattern. We found that the expression of all but two GST genes (IbGSTZ3 and $I b E F 1 B \gamma 2$ ) significantly changed (i.e., more than two-fold increase or decrease) under at least one of the stress treatments (Fig. 5b). The significantly changed GSTs were found in each of seven subfamilies, which suggests that GST genes are widely involved in responses to diverse abiotic stresses in sweet potato. Moreover, hierarchical clustering analysis indicated that the overall stress-response patterns of investigated GST genes were remarkably different in the aboveground and underground tissues (Fig. 6b).

It is well-known that $\mathrm{H}_{2} \mathrm{O}_{2}$ plays dual roles in plant stress-response system, as a stressor that causes the injury to biological macromolecules and a signal molecule that induces the expression of a series of defense genes $[44,45]$. The present study determined that with $\mathrm{H}_{2} \mathrm{O}_{2}$ treatment, the majority of GST genes were significantly upregulated (i.e., more than two-fold increase) in the underground tissues, whereas that of aboveground tissues varied (i.e., some were upregulated, some were downregulated, and some did not change (Figs. 5b and 6b). In particular, the most severely affected GSTs were found exclusively with $\mathrm{H}_{2} \mathrm{O}_{2}$ treatment [i.e., $\log _{2}$ (fold changes) $>6$; Fig. 5b]. These data indicate that a large number of sweet potato GST genes are involved and might play pivotal roles in stress-response pathways that are mediated or triggered by $\mathrm{H}_{2} \mathrm{O}_{2}$.

Arsenic and cadmium are two heavy metals that are usually toxic to plants. With arsenic and cadmium treatments, 12 and 14 GSTs, respectively, were significantly affected in the underground tissues. All but one gene were upregulated and 8 of these were common in both treatments, and most genes belong to the Tau subfamily (Fig. 5b). In contrast, distinct gene expression patterns were observed in the aboveground tissues (Fig. 5b). Copper and zinc are regarded as two necessary trace elements that cause stress when present at high concentrations in plants [46]. With copper treatment, a number of genes were significantly influenced, and most of the affected genes were downregulated. Two genes 


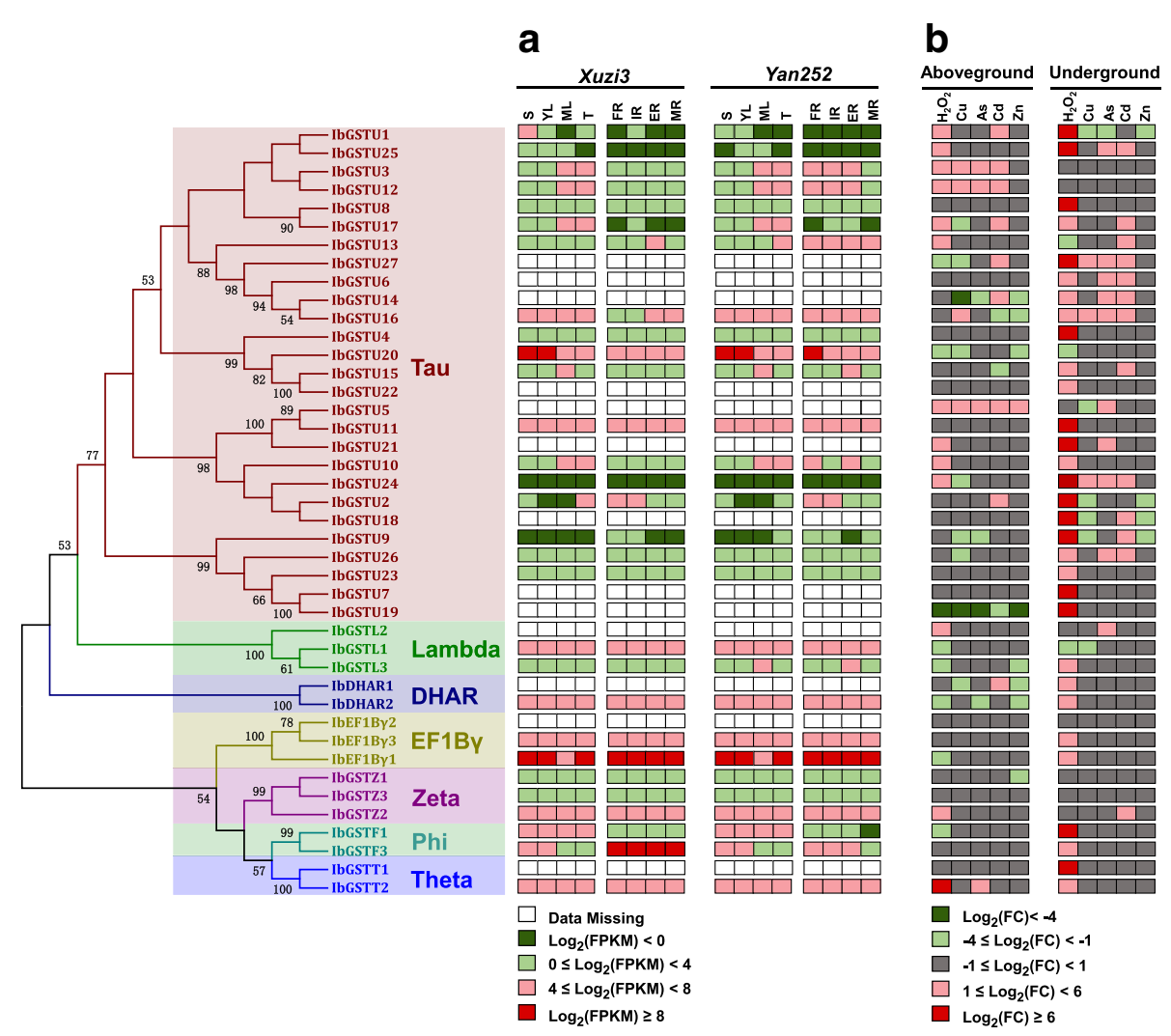

Fig. 5 Gene-expression profiles of sweet potato GSTs. a The FPKM data of 27 GST genes across 8 different tissues of purple-flesh and non-purple-flesh sweet potato were extracted from DB16 (log2 transformed, see text for more details) and shown (dark green, log2(FPKM) $<0$; light green, $0<\log 2$ (FPKM) $<4$; pink, $4<\log 2($ FPKM $)<8$; red, $\log 2($ FPKM $)>8$ ). DM, data missing; S, shoot; $Y L$, young leaf; $M L$, mature leaf; $T$, stem; FR, fibrous root; IR, initial tuberous root; ER, expending tuberous root; MR, mature tuberous root, respectively. $\mathbf{b}$ Gene-expression profiles of sweet potato GST genes in response to various abiotic stresses. The fold changes (test to control samples in aboveground and underground tissues, respectively) of gene expression were calculated and shown. The expression data of all 42 full-length GSTs were obtained by quantitative RT-PCR. $\mathrm{H}_{2} \mathrm{O}_{2}$, hydrogen peroxide; $\mathrm{Cu}$, copper; As, arsenic; Cd, cadmium; Zn, zinc, respectively

(IbGSTU14 \& IbGSTU19) were significantly downregulated in aboveground tissues (Fig. 5b). With zinc treatment, only one gene (IbGSTU5) was upregulated in the aboveground tissues; whereas several GSTs were downregulated, four in underground and nine in aboveground tissues (Fig. 5b).

Overall, the stress-response patterns of investigated GST genes substantially varied as a consequence of evolutionary diversification. Some GST genes are involved in the responses to multiple stressors, whereas some others respond to a specific stressor (Fig. $5 \mathrm{~b}$ ). In particular, in aboveground tissues, IbGSTU19 was downregulated and IbGSTU5 was upregulated in any treatment involving five abiotic stressors; IbGSTU3 3 and IbGSTU12 were upregulated after treatment with $\mathrm{H}_{2} \mathrm{O}_{2}, \mathrm{Cu}$, As, and $\mathrm{Cd}$. In underground tissues, IbGSTU24, IbGSTU27, and IbGSTU16 were upregulated after treatment with $\mathrm{H}_{2} \mathrm{O}_{2}, \mathrm{Cu}$, As, and Cd. Most of other GSTs responded to one or two stressors in either aboveground or underground tissues specifically. These results suggest that different GST members have been recruited and consequently divergent regulatory networks have been evolved in response to abiotic stresses in aboveground and underground tissues in sweet potato.

\section{Discussion}

Transcriptome-based gene family analyses in genetically complex organisms

Despite great advances in sequencing technologies, it remains costly and technically challenging to obtain highquality reference genomes of genetically complex organisms $[47,48]$.In this study, we report 43 full-length and 19 partial GST genes that were identified from local transcriptome databases in sweet potato, a hexaploid crop lacking a high-quality reference genome. Molecular cloning and Sanger sequencing successfully validated the existence of 42 full-length GST genes (i.e., $97.67 \%$ correctness) in a single sweet potato variety. These data highlight the high quality of our transcriptome databases, which could be used for characterization of gene 

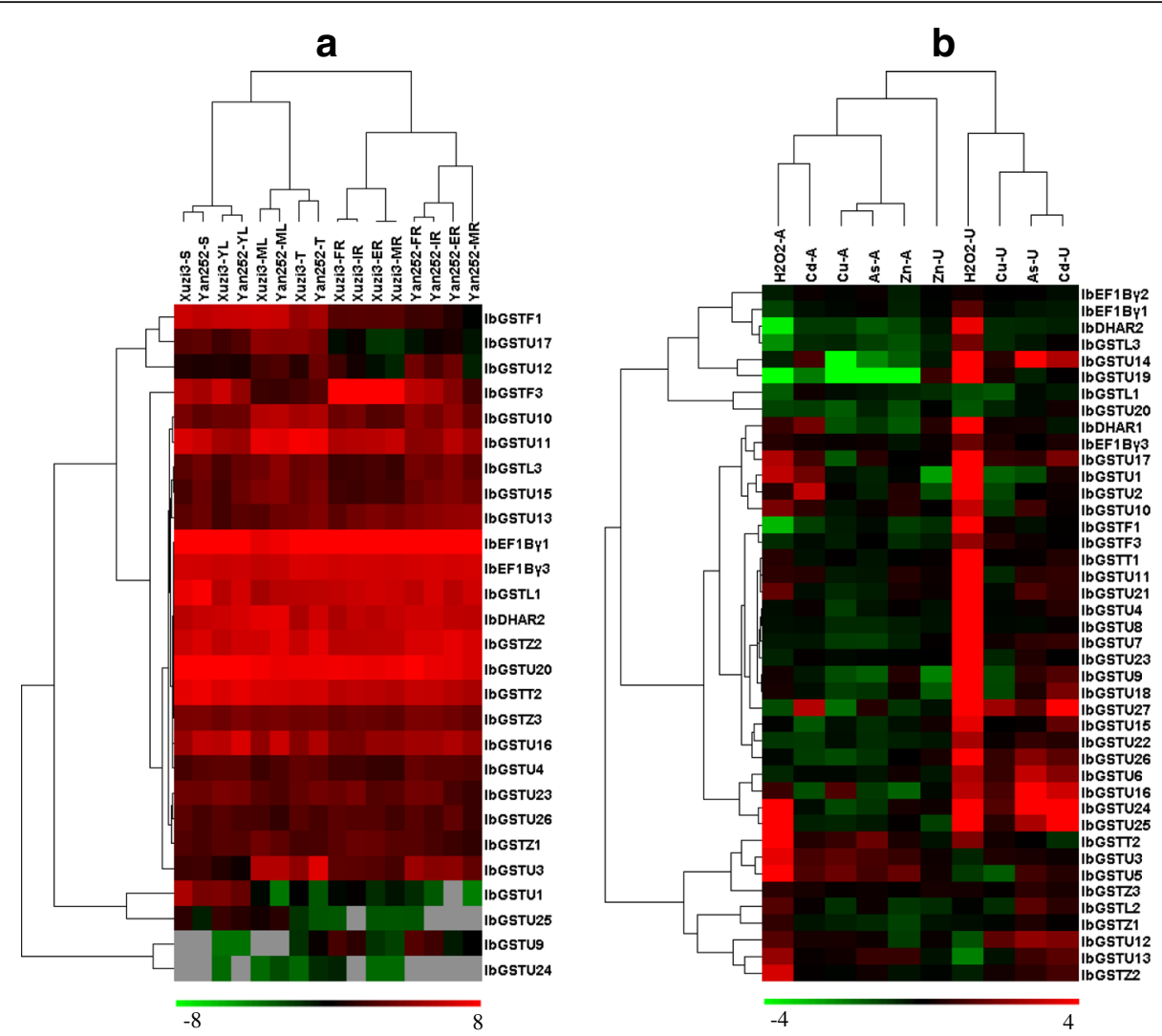

Fig. 6 Hierarchical clustering of gene-expression data. a The FPKM data of 27 GST genes across 8 different tissues of purple-flesh and non-purple-flesh sweet potato were extracted from DB16 (log2 transformed, see text for more details) and color scaled as shown in the bottom (gray boxes mean FPKM = 0). $\mathrm{S}$, shoot; $Y \mathrm{~L}$, young leaf; ML, mature leaf; $T$, stem; FR, fibrous root; IR, initial tuberous roots; ER, expending tuberous root; MR, mature tuberous root. $\mathbf{b}$ The fold changes (test to control samples in aboveground and underground tissues, respectively) of gene expression in five experiments of abiotic stress treatments were calculated and color scaled as shown in the bottom. The expression data of all 42 full-length GSTs were obtained by quantitative RT-PCR. - $A$, aboveground; $-U$, underground; $\mathrm{H}_{2} \mathrm{O}_{2}$, hydrogen peroxide; $\mathrm{Cu}$, copper stress; $\mathrm{As}$, arsenic; $\mathrm{Cd}$, cadmium; $\mathrm{Zn}$, zinc

families. To our knowledge, this is the first study characterizing a gene family in sweet potato, which could be applied to other genetically complex organisms without currently available genomic sequences.

RNA-seq is not only useful for gene discovery but also for quantifying transcript abundance, which could be applied to study the spatiotemporal profile of a gene and the pattern of gene-expression divergence of a gene family. In the present study, we investigated the gene-expression profiles of 42 full-length GST genes in the tuberous roots of 77 sweet potato varieties (the DB77 dataset) and 8 different tissues of each of two varieties (the DB16 dataset). These experiments have provided fundamental gene expression data of GST genes and revealed important insights into the evolution (especially in regulatory regions) of the GST gene family. Today, it becomes costly affordable to survey a relatively large number of samples using RNA-seq and thus could be easily applied to genetically complex organisms.

Although transcriptome-based gene family analyses are feasible and useful in genetically complex organisms, one should be aware of its limitations and cautious in data interpretation. Firstly, it might be difficult to collect all members of a gene family in a species because only expressed members could be possibly gathered by RNA-seq. This could be the main reason why we identified relatively less GSTs out of DB12 and DB77 than those of the sweet potato relatives (e.g., I. trifida and I. nil; Table 2). Incomplete identification of gene family members might produce a wrong or incomplete phylogenetic tree and lead to imprecise interpretations. Second, transcriptome-derived sequences neither contain information on regulatory ciselements nor exon-intron structures, which are important to infer the evolution of a gene family. Third, information on the locations of gene members on chromosomes is currently not available, which might hinder in investigations relating to genome evolution and speciation.

\section{Evolution and functional divergence of sweet potato GST genes}

After gene duplication, mutations in two duplicates could occur in either coding sequences or regulatory regions. 

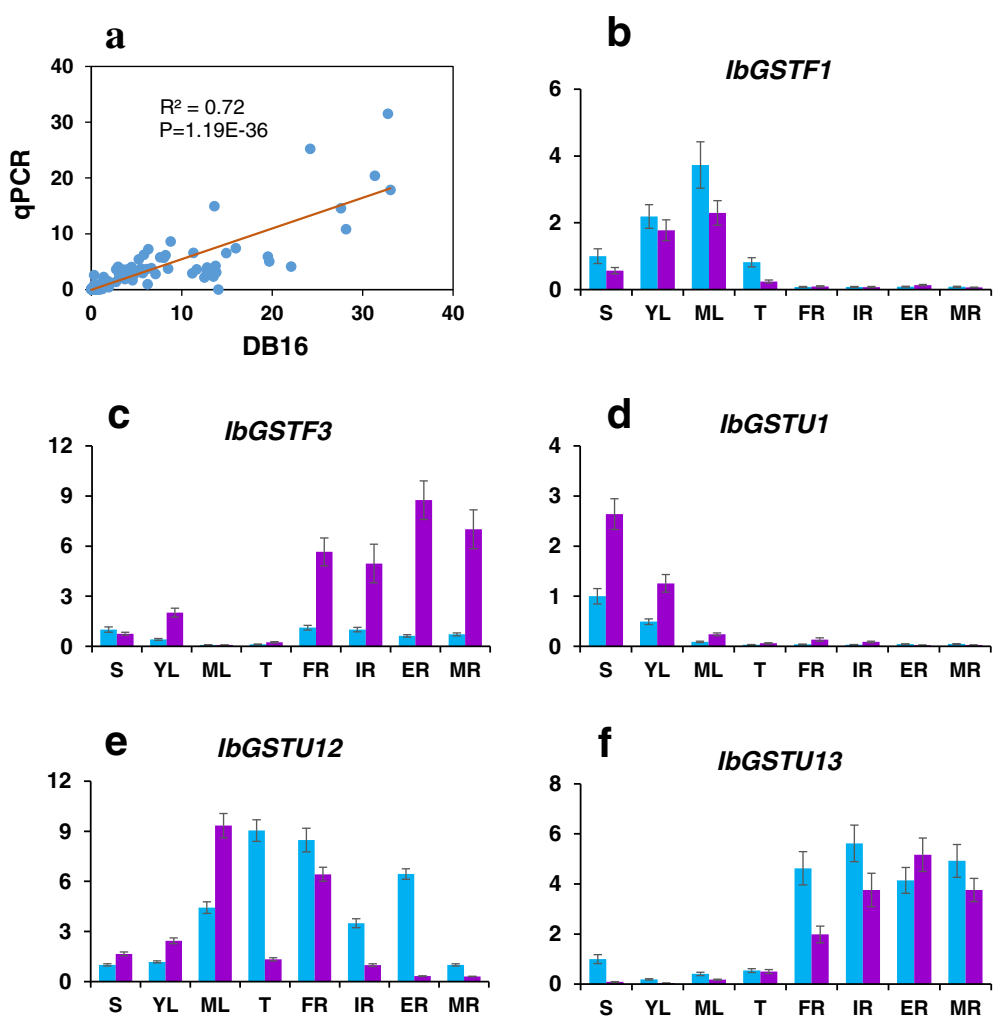

\section{IbGSTU15}

h IbGSTU2O
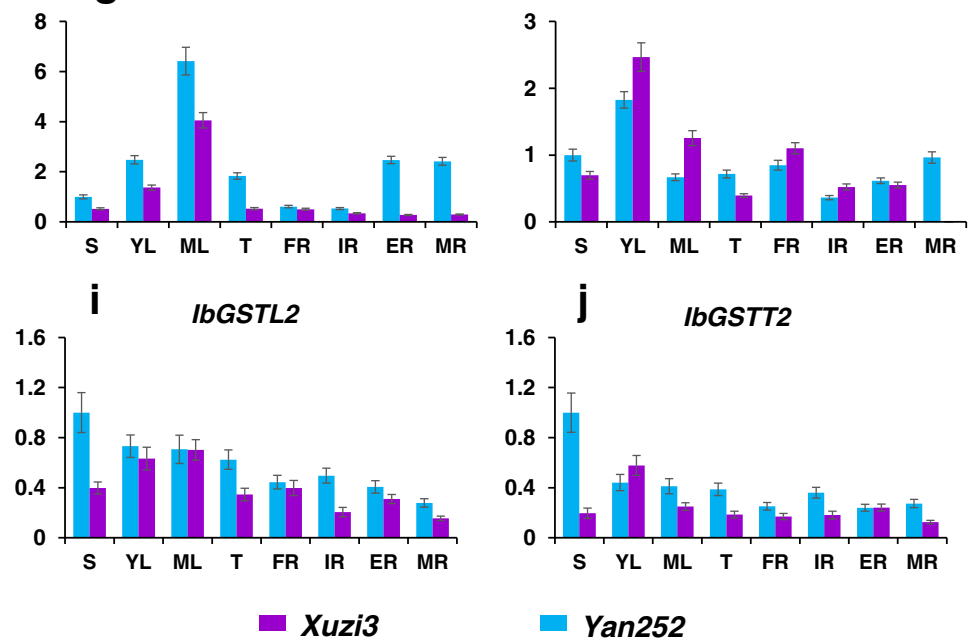

Fig. 7 Quantitative PCR analysis of 9 GST genes to validate their expression profiles obtained from DB16. a The Pearson's correlation between FPKM values obtained from DB16 and gene-expression data gained by qPCR. $R^{2}$ and $P$ value are shown. $\mathbf{b}$-j Relative expression of 9 GST genes were examined in two varieties of sweet potato, one is purple-flesh (Xuzi3) and the other is non-purple-flesh sweet potato (Yan252). For each sample, three duplicate PCR reactions were performed and the resulting data were used to calculate the mean and the standard error, which are shown in the panels. S, shoot; YL, young leaf; $M L$, mature leaf; $T$, stem; FR, fibrous root; IR, initial tuberous root; ER, expending tuberous root; MR, mature tuberous root

The former could modify protein properties and the latter might alter gene expression profiles spatiotemporally, both of which could result in functional divergence of duplicated genes $[2,49]$. In the present study, we investigated the divergence of 42 full-length sweet potato GSTs in both coding sequences and gene-expression profiles. Our analyses revealed an evolutionary pattern for GST genes across various species: largely conversed within and highly divergent among gene subfamilies. These results suggest that the main gene subfamilies in sweet potato were of 
ancient origin. However, signatures specific to recent gene duplications within a subfamily (at least after species divergence of the ancestor of sweet potato from that of A. thaliana) were also detected. This is consistent to available knowledge that sweet potato underwent multiple whole genome duplication events during its hexaploidization [32]. However, how different homeologs within each subfamily diverged after sweet potato speciation remains unclear. On the other hand, we demonstrated that the expression of a specific GST gene was dependent on tissues as well as genetic backgrounds, and remarkable divergence had occurred in gene expression profiles among different GST paralogs, i.e., substantial genetic differentiations might have accumulated in regulatory regions of studied GST genes, even in members within the same subfamily.

The question whether diversification of coding sequences and gene expression patterns in duplicated genes are correlated has been a topic of intense debate [50]. Fox example, Wagner et al. (2000) studied the relationship between expression profiles and protein sequence among yeast duplicate genes and found no significant correlation [51]. Makova et al. (2003) uncovered that nonsynonymous (Ka) and synonymous (Ks) substitution rates were significantly correlated with gene expression divergences of human duplicate genes at early stages after duplication [50]. McCarthy et al. (2015) reported that the functional divergence between two Arabidopsis paralogous genes is attributable to both regulation and changes in coding sequence [52]. In our study, we examined whether two genes with high similarity in coding sequences shared high similarity in gene expression patterns across different tissues. We found that most closely related genes showed different gene expression patterns. Based on our results, we postulate that divergence in coding sequences and regulatory regions of the two paralogous GST genes is uncoupled.

\section{Distinct GST-mediated networks in aboveground and underground tissues of sweet potato in response to abiotic stresses}

GST genes play important roles during plant growth, especially in plant defense or resistance to specific noxious chemicals. However, the function of each GST member, how the gene takes effect, and how the gene interplays each other remain unclear. In particular, related knowledge on the species sweet potato, a hexaploid crop with extraordinary capacity of adapting to different stressful environments such as high salinity, drought, and polluted soils, is limited. In the present study, we investigated the stress-response patterns of 42 sweet potato GST genes by the stress treatments of $\mathrm{H}_{2} \mathrm{O}_{2}, \mathrm{Cu}$, As, $\mathrm{Cd}$, and $\mathrm{Zn}$, respectively. Our data clearly exhibited divergences in the stress-response patterns of GST paralogs, which is in agreement with our observations from sequence analyses and gene-expression profiles. The majority of GST genes were specifically involved in response to one or two single stressors, whereas some GSTS responded to multiple abiotic stresses (e.g., IbGSTU5, IbGSTU19, IbGSTU24, and IbGSTU27). These results imply that the biological functions as well as the degree of importance of different GST paralogs were highly divergent in the whole stressresponse system in sweet potato and likely in other higher plants. In particular, almost all investigated GSTs showed distinct stress-response patterns between aboveground and underground tissues (Fig. 5b). Based on our results, we inferred that different GST-mediated networks involved in aboveground and underground tissues in response to abiotic stresses in sweet potato. In underground tissues, abiotic stresses caused by heavy metals and/or oxidizing agents (e.g., H2O2) would trigger signals, which subsequently activate specific GST genes (e.g., IbGST24, IbGSTU27, and IbGSTU16). Meanwhile, the signals would be transmitted to aboveground tissues where some other GST genes were activated (e.g., IbGSTU5 and IbGSTU12) or repressed (e.g., IbGSTU19). Further studies on how different GST members coordinate or interplay in the whole stress-response system are thus warranted.

\section{Conclusions}

In this study, we demonstrates the first example of transcriptome-based gene family analyses in sweet potato, a genetically complex and agronomically important crop. We identified and comparatively analyzed 42 full-length sweet potato GSTs in both coding sequences and gene-expression profiles, as well as their stress-response patterns. Our study systematically investigated the diversification of GST genes in sweet potato and provides useful information for elaborating the GST-mediated stress-response system in this worldwide crop as well as other plants.

\section{Additional files}

Additional file 1: Figure S1. Tissue sampling for DB12, DB16, and DB77. (DOCX $1990 \mathrm{~kb}$ )

Additional file 2: Table S1. Primers used in this study. (XLSX $15 \mathrm{~kb}$ ) Additional file 3: Table S2. Information of sweet potato GST genes identified in this study. (XLSX $59 \mathrm{~kb}$ )

Additional file 4: Table S3. Pairwise identity matrix for full-length GST genes in this study. (XLSX $22 \mathrm{~kb}$ )

Additional file 5: Figure S2. Phylogenetic relationships of 42 sweet potato GST proteins. (DOCX $41 \mathrm{~kb}$ )

Additional file 6: Table S4. Information of GSTs from other species used in this study. (XLSX $39 \mathrm{~kb}$ )

Additional file 7: Figure S3. Phylogenetic tree and subfamily classification of GST proteins from sweet potato, Ipomoea nil, Ipomoea trifida, and Arabidopsis thaliana. (DOCX $1419 \mathrm{~kb}$ )

Abbreviations

FPKM: Fragments per kilobase of transcript per million fragments mapped; GST: Glutathione S-transferase; RNA-seq: whole transcriptome shotgun sequencing 


\section{Acknowledgements}

This study was jointly supported by the Priority Academic Program Development of Jiangsu Higher Education Institutions; National Natural Science Foundation of China (Grant No. 31771855); Natural Science Foundation of Jiangsu Province (Grant No. BK20141146); National Key Laboratory of Plant Molecular Genetics (Grant No. Y409Z111U1).

\section{Funding}

The Priority Academic Program Development of Jiangsu Higher Education Institutions; National Natural Science Foundation of China (Grant No. 31771855); Natural Science Foundation of Jiangsu Province (Grant No. BK20141146); National Key Laboratory of Plant Molecular Genetics (Grant No. Y409Z111U1).

\section{Availability of data and materials}

The raw data of RNA-seq experiments generated in this study have been deposited in the Genome Sequence Archive of Beijing Institute of Genomics, Chinese Academy of Sciences (the accession numbers of DB12, DB16, and DB77 are CRA000288, CRA000606, and CRA000608, respectively). The datasets of DB12 and DB77 analyzed during the current study are available from the corresponding author on reasonable request.

\section{Authors' contributions}

$Y L$ designed the research. ND and YL analyzed the data and wrote the manuscript with contributions from other coauthors. All coauthors contributed to the experiment and data analysis, and approved the final manuscript.

\section{Ethics approval and consent to participate} Not applicable.

\section{Consent for publication}

Not applicable.

\section{Competing interests}

The authors declare that they have no competing interests.

\section{Publisher's Note}

Springer Nature remains neutral with regard to jurisdictional claims in published maps and institutional affiliations.

Received: 24 July 2017 Accepted: 17 November 2017

Published online: 28 November 2017

\section{References}

1. Stirnimann CU, Petsalaki E, Russell RB, Müller CW, Chaudhuri I, Söding J, et al. Evolution by gene duplication: an update. 2003.

2. Raes J. Duplication and divergence: the evolution of new genes and old ideas. Annu Rev Genet. 2004;38(1):615-43.

3. Li WH, Yang J, Gu X. Expression divergence between duplicate genes. Trends Genet. 2005;21(11):602-7.

4. Lan T, Yang ZL, Xue Y, Liu YJ, Wang XR, Zeng QY. Extensive functional diversification of the Populus glutathione S-transferase supergene family. Plant Cell. 2009;21(12):3749-66

5. Dixon DP, Adrian L, Robert E. Plant glutathione transferases. Genome Biol. 2002;401(3):169-86.

6. Sheehan D, Meade G, Foley VM, Dowd CA. Structure, function and evolution of glutathione transferases: implications for classification of non-mammalian members of an ancient enzyme superfamily. Biochem J. 2001;360:1-16.

7. Mohsenzadeh S, Esmaeili M, Moosavi F, Shahrtash M, Saffari B, Mohabatkar H. Plant glutathione S-transferase classification, structure and evolution. Afr J Biotechnol. 2011;10(42):8160-5.

8. Mannervik B. Glutathione transferase. Annu Rev Pharmaco. 2010;8(1):e0131.

9. Edwards R, Dixon DP, Walbot V. Plant glutathione S-transferases: enzymes with multiple functions in sickness and in health. Trends Plant Sci. 2000;5(5):193-8.

10. Marrs KA. The functions and regulation of glutathione s-transferases in plants. Annu Rev Plant Physiol Mol Biol. 1996:47(47):127.

11. Agrawal GK, Jwa NS, Rakwal R. A pathogen-induced novel rice (Oryza sativa L.) gene encodes a putative protein homologous to type II glutathione $\mathbf{S}$ -transferases. Plant Sci. 2002;163(6):1153-60.
12. Kampranis SC, Damianova R, Atallah M, Toby G, Kondi G, Tsichlis PN, et al. A novel plant glutathione S-transferase/peroxidase suppresses Bax lethality in yeast. J Biol Chem. 2000;275(38):29207-16.

13. Loyall L, Uchida K, Braun S, Furuya M, Frohnmeyer $H$. Glutathione and a UV light-induced glutathione S-transferase are involved in signaling to chalcone synthase in cell cultures. Plant Cell. 2000;12(10):1939-50.

14. Dixon DPS. Mark, Edwards R. Roles for glutathione transferases in plant secondary metabolism. Phytochemistry. 2010;71(4):338-50.

15. Bianchi MW, Roux C, Vartanian N. Drought regulation of GST8, encoding the Arabidopsis homologue of ParC/Nt107 glutathione transferase/peroxidase. Physiol Plantarum. 2002;116(1):96-105.

16. Deridder BP, Dixon DP, Beussman DJ, Edwards R, Goldsbrough PB. Induction of glutathione S-Transferases in Arabidopsis by herbicide safeners. Plant Physiol. 2002;130(3):1497-505.

17. Ryu HY, Kim SY, Park HM, You JY, Kim BH, Lee JS, et al. Modulations of AtGSTF10 expression induce stress tolerance and BAK1-mediated cell death. Biochem Bioph Res Co. 2009;379(2):417-22.

18. Li ZS, Alfenito M, Rea PA, Walbot V, Dixon RA. Vacuolar uptake of the phytoalexin medicarpin by the glutathione conjugate pump. Phytochemistry. 1997;45(4):689-93.

19. Goodman CD, Walbot V. AN9, a petunia glutathione S-Transferase required for anthocyanin sequestration, is a flavonoid-binding protein. Plant Physiol. 2000;123(4):1561-70.

20. Conn S, Curtin C, Bézier A, Franco C, Zhang W. Purification, molecular cloning, and characterization of glutathione S-transferases (GSTs) from pigmented Vitis vinifera L. cell suspension cultures as putative anthocyanin transport proteins. J Exp Bot. 2008;59(13):3621-4.

21. Marrs KA, Alfenito MR, Lloyd AM, Walbot V. A glutathione S-transferase involved in vacuolar transfer encoded by the maize gene Bronze-2. Nature. 1995:375(6530):397-400

22. Larsen ES, Alfenito MR, Briggs WR, Walbot V. A carnation anthocyanin mutant is complemented by the glutathione $S$-transferases encoded by maize Bz2 and petunia An9. Plant Cell Rep. 2003;21(9):900.

23. Board PG, Baker RT, Chelvanayagam G, Jermiin LS. Zeta, a novel class of glutathione transferases in a range of species from plants to humans. Biochem J. 1997:328(Pt 3):929-35.

24. Thom R, Dixon DP, Edwards R, Cole DJ, Lapthorn AJ. The structure of a zeta class glutathione S-transferase from Arabidopsis thaliana: characterisation of a GST with novel active-site architecture and a putative role in tyrosine catabolism. J Mol Biol. 2001;308(5):949-62.

25. Dixon DP, Davis BG, Edwards R. Functional divergence in the glutathione transferase superfamily in plants. Identification of two classes with putative functions in redox homeostasis in Arabidopsis thaliana. J Biol Chem. 2002. 277(34):30859-69.

26. Dixon DP, Edwards R. Roles for stress-inducible lambda glutathione transferases in flavonoid metabolism in plants as identified by ligand fishing. J Biol Chem. 2010;285(47):36322-9.

27. Vickers TJ, Wyllie S, Fairlamb AH. Leishmania major elongation factor $1 \mathrm{~B}$ complex has trypanothione S-transferase and peroxidase activity. J Biol Chem. 2004;279(47):49003-9.

28. He G, Guan CN, Chen QX, Gou XJ, Liu W, Zeng QY, et al. Genome-wide analysis of the GlutathioneS-Transferase gene family in Capsella rubella: identification, expression, and biochemical functions. Front Plant Sci. 2016;7(e0131):1325.

29. Woolfe JA. Sweet potato: an untapped food resource. 1992.

30. Magoon ML, Krishnan R, Bai KV. Cytological evidence on the origin of sweet potato. Theor Appl Genet. 1970;40(8):360-6

31. Oziasakins P, Jarret RL. Nuclear-DNA content and ploidy levels in the genus ipomoea. J Am Soc Hortic Sci. 1994;119(1):110-5.

32. Yang J, Moeinzadeh M, Kuhl H, Helmuth J, Xiao P, Liu G, et al. The haplotype-resolved genome sequence of hexaploid Ipomoea batatas reveals its evolutionary history. bioRxiv. 2016:064428. doi: 10.1101/064428.

33. Mortazavi A, Williams BA, Mccue K, Schaeffer L, Wold B. Mapping and quantifying mammalian transcriptomes by RNA-Seq. Nat Methods. 2008;5(7):621.

34. Wang Z, Gerstein M, Snyder M. RNA-Seq: a revolutionary tool for transcriptomics. Nat Rev Genet. 2009;10(1):57-63.

35. Eid J, Fehr A, Gray J, Luong K, Lyle J, Otto G, et al. Real-time DNA sequencing from single polymerase molecules. Science. 2009;323(5910):133-8.

36. Gunel M. Next-generation DNA sequencing. 2010.

37. Koren S, Schatz MC, Walenz BP, Martin J, Howard J, Ganapathy G, et al. Hybrid error correction and de novo assembly of single-molecule sequencing reads. Nat Biotechnol. 2012;30(7):693-700. 
38. Sharon D, Tilgner $H$, Grubert F, Snyder M. A single-molecule long-read survey of the human transcriptome. Nat Biotechnol. 2013;31(11):1009-14.

39. Luo Y, Ding N, Shi X, Wu Y, Wang R, Pei L, et al. Generation and comparative analysis of full-length transcriptomes in sweetpotato and its putative ancestor. bioRxiv. 2017:112425. doi: 10.1101/112425.

40. Hall TA. BioEdit: a user-friendly biological sequence alignment editor and analysis program for windows 95/98/NT. Nucl Acids Symp Ser. 1999;41:95-8.

41. Kumar S, Stecher G, Tamura K. MEGA7: molecular evolutionary genetics analysis version 7.0 for bigger datasets. Mol Biol Evol. 2016;33(7):1870.

42. Wagner U, Edwards R, Dixon DP, Mauch F. Probing the diversity of the Arabidopsis glutathione S-Transferase gene family. Plant Mol Biol. 2002;49(5):515-32.

43. Levin JZ, Yassour M, Adiconis X, Nusbaum C, Thompson DA, Friedman N, et al. Comprehensive comparative analysis of strand-specific RNA sequencing methods. Nat Methods. 2010;7(9):709.

44. Neill S, Desikan R, Hancock J. Hydrogen peroxide signaling. Curr Opin Plant Biol 2002; 5(5):388-395.

45. Veal EA, Day AM, Morgan BA. Hydrogen peroxide sensing and signaling. Mol Cell. 2007;26(1):1.

46. Singh S, Parihar P, Singh R, Singh VP, Prasad SM. Heavy metal tolerance in plants: role of transcriptomics, proteomics, metabolomics, and ionomics. Frontiers Plant Sci. 2015;6:1143.

47. Dufresne F, Stift M, Vergilino R, Mable BK. Recent progress and challenges in population genetics of polyploid organisms: an overview of current state-ofthe-art molecular and statistical tools. Mol Ecol. 2014;23(1):40-69.

48. Manuel S, Martis MM, Matthias P, Thomas N, Mayer Klaus FX. Analysing complex Triticeae genomes-concepts and strategies. Plant Methods. 2013;9(1):1-9.

49. Zhang J. Evolution by gene duplication: an update. Trends Ecol Evol. 2003; 18(6):292-8.

50. Makova KD, Li WH. Divergence in the spatial pattern of gene expression between human duplicate genes. Genome Res. 2003;13(7):1638-45.

51. Wagner A. Decoupled evolution of coding region and mrna expression patterns after gene duplication: implications for the neutralist-selectionist debate. P Nat Acad Sci USA. 2000;97(12):6579-84.

52. Mccarthy EW, Abeer M, Amy L. Functional divergence of APETALA1 and FRUITFULL is due to changes in both regulation and coding sequence. Front Plant Sci. 2015;6:1076.

\section{Submit your next manuscript to BioMed Central and we will help you at every step:}

- We accept pre-submission inquiries

- Our selector tool helps you to find the most relevant journal

- We provide round the clock customer support

- Convenient online submission

- Thorough peer review

- Inclusion in PubMed and all major indexing services

- Maximum visibility for your research

Submit your manuscript at www.biomedcentral.com/submit

) Biomed Central 